\title{
Cost Analysis of Medications Used in Upper Respiratory Tract Infections and Prescribing Patterns in University Sans Malaysia, Penang, Malaysia
}

\author{
Ahmad Abdulrahman Almeman ${ }^{1 *}$, Mohamed Izham Mohamed Ibrahim $^{2}$ and \\ Seemab Rasool ${ }^{3}$ \\ ${ }^{1}$ Pharmacy School-Uniazah, Almulaida, Qassim University, Kingdom of Saudi Arabia, ${ }^{2}$ College of Pharmacy, Qatar University, \\ Qatar, ${ }^{3}$ Prince Sultan Cardiac Center, Buraidah , Qassim , Kingdom of Saudi Arabia
}

*For correspondence: Email: ahmadalmeman@gmail.com; Tel: 00966548889712; Fax: 0096663800662

\begin{abstract}
Purpose: To conduct a cost analysis, a narrow cost-utility study, for upper respiratory tract infection medications in University Sans Malaysia's clinics.

Methods: Retrospective analysis was done for all medical claims of upper respiratory tract infections in the period 2008 - 2009. The study was done in the clinics under University Sains Malaysia. A total of 302 patients suffering from upper respiratory tract infection were enrolled. Cost analysis of the pattern of antibiotic prescriptions for upper respiratory tract infections was done. For cost analysis, costs were calculated based on the acquisition cost from the perspective of the medical center (USM) as the provider.

Results: The research results states that there were 90\% among the patients were prescribed an antibiotic, 81 percent were prescribed an antihistamine, 81 percent were prescribed an analgesic, and 21 percent were prescribed a mucolytic or an expectorant.

Conclusion: In comparison to the previously revised researchers, the percentage of the prescribed antibiotics is very high which is unacceptable. A tremendous amount of expenditure and other adverse events can be avoided by halting this behavior. A local guideline for prescribing antibiotics and implementing the same is the need of the hour.
\end{abstract}

Keywords: Prescribing patterns, Cost analysis, Upper respiratory tract, Infections, Antibiotics

Tropical Journal of Pharmaceutical Research is indexed by Science Citation Index (SciSearch), Scopus, International Pharmaceutical Abstract, Chemical Abstracts, Embase, Index Copernicus, EBSCO, African Index Medicus, JournalSeek, Journal Citation Reports/Science Edition, Directory of Open Access Journals (DOAJ), African Journal Online, Bioline International, Open-J-Gate and Pharmacy Abstracts

\section{INTRODUCTION}

Upper respiratory infections (URTIs) are the most common seasonal infections which include pharyngitis, sinusitis, epiglottitis, laryngotracheitis, and the common cold. Viruses play the most significant role in the pathogenesis of most of these infections [1]. Typically, $70-80 \%$ of URTIs are triggered by viruses. However, 70 $80 \%$ of patients worldwide are being prescribed antibiotics that are inconsistent with the causative organism. Thus, it is essential to differentiate between bacterial and viral infections to determine whether or not to give antibiotics.

Ideally, viruses do not require any type of treatment when they infect the upper respiratory tract [2]. It has been recognized that the problem of antibiotic abuse is a result of a complex interplay of various socio-cultural, economic and cognitive factors at the level of the patients, the 
prescribers and the drug industry [3]. Most studies have shown that inappropriate prescribing for upper respiratory tract infections is the norm with significant cost and health consequences [4]. The high cost of drugs, particularly those used to treat URTIs, is an increasing important concern for health institutions worldwide. Moreover, the misuse of antibiotics has led to the development of antibiotic-resistant bacteria [2].

It is extremely difficult to approach and convince physicians by simple means such as knowledge sharing, pamphlet distribution, or medical alerts as these will not be helpful to change the current practices. Very few studies have been conducted to find the prescribing pattern of antibiotics in Malaysia. In 1999, Teng et al found that antibiotic was prescribed in $68.4 \%$ of encounters with URTI by general practitioners in a study conducted on private clinics in Malaysia [5]. In another study conducted on Antibiotic usage in pediatric respiratory tract infection on just 40 subjects the antibiotic prescription was found to be $70 \%[6]$.

The high cost of drugs, particularly those used to treat upper respiratory tract infections, is an increasingly important problem for health institutions worldwide. Thus, this study was carried out to conduct a cost analysis, i.e., a narrow cost-utility study, for upper respiratory tract infection medications in clinics at the University Sans Malaysia, Malaysia.

\section{METHODS}

\section{Setting}

The study was conducted in the Outpatient Department clinics under the jurisdiction of University Sans Malaysia (USM), Penang, Malaysia.

\section{Study design}

For clinical evaluation, a retrospective analysis for all medical claims of upper respiratory tract infections in the period January 2008 to December 2009 was analyzed and then compared to the global consensus guidelines. The ethical approval for the study was obtained from the University Sans Malaysia (USM), to which the clinics belong. The privacy of the patients and clinics was ensured before commencing this study.

\section{Inclusion and exclusion criteria}

All consultations for acute (Upper respiratory tract infections) URTIs were identified. The database is available physically in packs and we included all the patients who had an episode of URTIs in that period, regardless the specific diagnosis. Excluded were illegible claims (prescriptions) to avoid calculating a cost for wrong medication. Details of antibiotic therapy prescribed at the first or any subsequent consultations were recorded, including the drug, dose, frequency and duration of treatment to allow calculation of the cost of drugs prescribed. If the diagnosis was not known by the physician, the lay term "common cold" was used to represent generalized URTIs. To be as inclusive as much as possible for all medications prescribed for URTIs, yet to limit the inclusion of medications that would not be expected to be prescribed for URTIs, the analysis included only the following drug classes: antibiotics, antihistamines, and symptomatic medications (analgesics, bronchodilators, and inhaled corticosteroids). Combination antihistamine and decongestant medications are classified as symptomatic medications because of the effect of the decongestant. Topical or ophthalmic medications were excluded, so were medications such as antifungal, antibiotics, anthelmintics, and antiprotozoan agents.

\section{Cost analysis}

For cost analysis, costs were calculated based on the acquisition cost from the perspective of the medical center (USM) as the provider. Therefore, the cost of the medications was obtained from the USM's pricing lists and then used for the calculations. The net cost of the prescription is the collective costs of the medications in that prescription, added to the cost of the consultation. The cost of the consultation was fixed for each prescription, whereas the cost of medications varied according to the diagnosis and treating physician.

\section{Statistical analysis}

SPSS version 13 software was used to analyze the data. Descriptive analysis was used to summarise the pattern of utilization of antibiotics.

\section{RESULTS}

In total, 302 patients were included. Six claims were excluded due to illegibility. Clinical details of the infections in the records were not standardized and did vary significantly. The 
majority of patients were university staff or their relatives (wife, husband, son, daughter). Their ages ranged from 1 - 58 years, and among which 245 were male and 47 female.

\section{Drug prescriptions}

Notably, $90 \%$ among the patients were prescribed an antibiotic, 81 percent were prescribed an antihistamine, 81 percent were prescribed an analgesic, and 21 percent were prescribed a mucolytic or an expectorant.

The majority of the physicians did not include the exact diagnosis (only URTI). A notable proportion of visits included filled prescriptions for antibiotics, antihistamines, analgesic or expectorants. Among the antibiotics, erythromycin was the most popular (54\%) followed by amoxycillin (27\%). Doxycyclin, ampicillin, and cephalexin were prescribed also, however, in lower rates of 12,6 and $1 \%$, respectively.

For symptomatic treatment, the majority of the patients had prescriptions for antihistamine and analgesic. Paracetamol was used in all the clinics to control the pain-related symptoms. Rarely, mefanemic acid was used, especially in case of frequent headaches.

Among the prescribed antihistamines, loratidine was the only non-sedative agent and was prescribed for only 1 percent out of the patients.

Oppositely, Actifed $囚$, which has sedation as a side effect was prescribed for the majority of the patients.

\section{Cost estimates}

Erythromycin was prescribed the highest among them. The cost of erythromycin was $0.3 \mathrm{RM}(1$ Ringitt Malaysian $=0.09$ US dollars (USD)) per dose and was administered four times a day for three days. Thus, the net cost was 3.6 RM (1.12 USD) per patient. The cost of Amoxicillin was 0.4 RM (0.12 USD) per dose and was given three times a day for three days. The net cost was found to be 3.6 RM, too (1.12 USD). The cost of Doxycycline was 0.06 RM (0.02 USD) per dose and was dosed three times a day for five days. The net cost was 0.9 RM (0.28 USD) per patient. The cost of ampicillin was 0.14 RM (0.04 USD) per dose and was dosed three times a day for three days. The net cost was $3.1 \mathrm{RM}$ (1.0 USD) per patient. The cost of cephalexin was 0.21RM (0.07 USD) per dose and was dosed two times a day for five days. The net cost was 2.1 RM (0.7 USD) per patient.
Comparing the antihistamines, chlorpheniramine was the cheapest $(0.01 R M=0.03$ USD), followed by $A c t i f e d(R)$ (triprolidine + pseudoephidrine) (0.19 RM = 0.06 USD), and promethiazine (0.28 $\mathrm{RM}=0.09$ USD).

\section{DISCUSSION}

Over 200 different viruses have been isolated in patients with URIs [7]. Viruses account for up to $90 \%$ cases of URTIs. Generally, most studies show that inappropriate prescribing of antibiotics for upper respiratory tract infections is the norm and not the exception, with significant cost and health consequences [4].

It is essential to understand the global figures of antibiotic expenditure on direct costs to be able to evaluate and estimate the magnitude of costsaving possible. Despite predominant viral etiology, 50 to $75 \%$ patients are inappropriately being prescribed antibiotics globally [8]. The situation in Malaysia is that antibiotics are prescribed in $90 \%$ cases of URTIs. This percent is even much higher than global figures and more control should be applied [9].

The results of this study showed that we have a very high rate of antibiotic prescription. Importantly, in addition to the high antibiotic prescription, we the prescription of antibiotics was not according to guidelines. It could be justified by the availability and or accessibility to these drugs. For instance, if macrolides were not available in that day, amoxicillin was prescribed as an alternative. If both had been depleted already, doxycyclin, ampicillin, and cephalexin were prescribed.

Most of the published data and guidelines have different figures than ours, antibiotics are used for viral infections between 15 and $72 \%$ of the time $[5,6,8]$. Oral penicillin or erythromycin (in penicillin-allergic persons), given for 10 days, remains the preferred agent for GABHS-related pharyngitis [10]. Oral amoxicillin, trimethoprimsulfamethoxazole, or doxycyclin, given for 3 to 10 days, are the recommended first-line antibiotics for the treatment of moderate to severe acute sinusitis [11]. From point of view of cost, Doxycyclin is the cheapest antibiotic and always should be considered as the first line therapy. On the other hand, clinically, amoxicillin and Ampicillin are the most effective regimen if the URTI was due to bacterial origins. In our situation, neither was considered to be the first line therapy. Moreover, ampicillin is better and cheaper than erythromycin. Obviously, the 
mismanagement of antibiotic usage can be detected easily.

Another issue is that doxycyclin has the most tendencies to develop gasteroentestinal adverse events among the majority of the patients. On the other hand, this can be outweighed by its tiny cost comparing to others. Thus, someone may consider it as the potential second line therapy if amoxicillin is not accessible. Cephalexin is now one of the best choices for URTI due to its easiness of administration, twice a day, cost, and its tolerability. Furthermore, Cephalexin is a specific antibiotic which can kill less useful bacteria comparing to the broad spectrum ones such as amoxicillin, doxycyclin, ampicillin, and erythromycin. Accordingly, in terms of future resistance, cephalexin has perhaps the lowest tendency among all. Then even if the resistance develops, Cephalexin is a narrow spectrum antibiotic which is rarely prescribed in serious diseases and is easily replaced by other alternatives [12].

By having the awareness increased regarding the viral infections, the majority of these costs can be reduced, saving the patients' and healthcare providers' budget.

Antibiotics are well known to cause harms at several degrees. At the level of URTI, the utilized antibiotics may cause gastrointestinal disturbances, headache, and colon related problems. In these cases patients come back to the treating physicians and require more medications or physicians will prescribe the required medications in combination at the first visit. The second is more common in our situation. Both scenarios contribute to the overall costs and may duplicate the net cost per visit. Moreover, sometimes the prescription of a second antibiotic is given which increases the cost of treatment of URTIs, even though neither the first nor the second antibiotic was indicated. Accordingly, a substantial proportion of resources in the management of URTIs is being allocated to unnecessary and useless treatments. This finding is particularly important in a government funded health system like oncampus GPs because of the competing priorities for money.

Furthermore, the issues of medication wastage and unused medications have been a problem in this setting. It was shown that $15 \%$ of the prescribed medications are not used totally, and $84 \%$ are used only partially. Moreover, 3\% among which will expire before utilization. The accumulated cost for all the wastages was estimated to be $47 \%$ of the expenditure [13]. Our study didn't look at the wastages, but was conducted on the same campus.

In case of some particular antibiotics, if combined with non-appropriate prescription, serious neurological and haematological symptoms may occur which require admission and emergency treatment. Although rare, if it happens, it will raise the cost significantly not only at the level of the general practitioner, but rather at the level of general hospitals. One of these serious events is called antibiotic-induced-colitis which results from the excessive uses of antibiotics that in turn induce imbalance of the normal flora of the colon. Considering the other finding, a notable proportion of visits included filled prescriptions for a combination of an antibiotic, antihistamine, analgesic and expectorant, USM should identify the government policy towards the antibiotics, restrictions on the purchase, and so on. It is possible that there is no regulation controlling purchase of the antibiotics and the citizens purchase antibiotics with the strong belief that they would be cured very quickly. At the same time, it is necessary to identify if there are any advertisements promoting antibiotics or there is a restriction for advertisement of this type.

Clinically, expectorants have very minimal contribution to overcome the signs and symptoms. Therefore, most of the clinical guidelines voted against using them unless the respiration has been compromised significantly [14]. On the other hand, leaving the sputum expectorated naturally will not harm in the majority of the URTIs. In our case, only about 5 percent of the patients were prescribed an expectorant or a mucolytic or in combination. This can be perceived as the awareness of the general practitioner regarding their uses.

As regards to mucolytics alone or in combination, there is no clinical benefits in such infections and therefore their usage should be ceased and saved for other severe lower respiratory infections.

The usage of the rest of the medications is justifiable and very reasonable. Obviously, they have been utilized only when necessary as they only represent in total of 3 percent.

As expected, from other published data, considering the contribution of each group to the total cost of URTIs, antibiotics have the highest contribution 58\%. In details, 163 patients were prescribed erythromycin at a cost of $3.6 \mathrm{RM}$ (1.12 USD) per prescription. The net cost is 3.6 multiplied by 163 equals to 586 RM (182.4 USD). Similarly, the net costs are 291 RM (90.5 USD), 
32.4 RM (10 USD), 57 RM (17.7 USD), and 6.3 RM (1.96 USD) for amoxicillin, doxycyclin, ampicillin, and cephalexin; respectively. The net costs for all the prescribed antibiotic is $972.7 \mathrm{RM}$ (303 USD) for 302 patients. Given that $75 \%$ of then should not have been prescribed antibiotics, thus we could have saved roughly $729.525 \mathrm{RM}$ (227 USD) for the 302 patients.

Antihistamine and analgesic contributions are 24 and $10 \%$, respectively, out of the total cost. Therefore, they contribute roughly to about 569 RM (182.4 USD) or 1.88 RM (0.585 USD) per prescription. This is very predictable, since they are the mainstay approach of the symptomatic therapy. Antihistamine's cost is higher than analgesic's cost because the using of Loratidine in some situation (1.02RM (0.32 USD)/tab). The use of this agent is strongly needed when the patient has to do particular works (e.g. driving, running machines) to avoid the accompanied risks of getting drowsy with old generation antihistamines [15]. Actually, this is supposed to be the net cost per prescription for the majority of the patients as the treatment of viral infections is symptomatic treatment solely. Since, the cost of loratidine was the most expensive one (1.02 $\mathrm{RM}$ ), thus it might not have be available widely in the clinics.

Actifed $\AA$ contains a substance that causes systemic vasoconstriction. There have been several warnings from researches to minimize its usage or even cease it especially with the presence of other safer alternatives. In addition, given the high concern of the government regarding addiction, Actifed $\AA$ can be abused and addicted easily and cheaply. It is widely available and easily accessible every where even within campus.

Corticosteroids contributed to $1 \%$ of the price, although, 9 items only were prescribed. Corticosteroids' inhalers have the highest cost per item among all the drugs (8 - 22 RM/item). Sometimes the patient will required to be given a bronchodilator agent such as salbutamol. However, it is a necessity when required and cannot be replaced or overlooked. Thus, this cost cannot be saved and should be considered when allocating the budget. Expectorants and mucolytics contributed to $5 \%$ out of the total cost, thus roughly about 83 RM (26 USD) for each 302 patients. Although is very minimal, it will be very significant when considering the whole country and population. One should think that expectorants and mucolytics are not proven to be effective in such situations. This was another reason for the increased cost/visit.

Accordingly, a substantial proportion of resources in the management of URTIs is being allocated to unnecessary and useless treatments. Overall, the net cost that could have been saved includes the sum of 729.525 RM (over-used antibiotics) and 83 (expectorants and mucolytics) which equals to 812 RM (253 USD). This figure accounts for almost 50 percent of the total cost of the URTIs.

Indirect costs for upper respiratory drug infections can be estimated by including the following potential costs to the patient: prescription charges transport costs and cost of time in travelling to and from the surgery and/or pharmacy together with the time spent at these places [16].

The practice of prescribing antibiotics for URTIs has notable implications beyond the fact that the antibiotics are ineffective and not-indicated treatments. Clinically, if the patient receives an antibiotic several times to treat the same bacteria or organism, resistance may develop. So, another intangible concerning cost is the cost of resistance. Having said that, the cost of next alternative is usually higher than the cost of the first choice, which in turn would add further financial burden to the total health cost. Furthermore, cross tolerance between different groups of antibiotics may emerge, leading to a persistent resistance and loss of lives.

Currently, several publications have reported the presence of such resistant bacteria among young school children and elderly $[17,18]$. This in turn reduces the compliance and trust of the patients, leading to more missed doses when medications are necessary. Evidence exists to indicate that the prevalence of drug-resistant bacteria is increasing. Tetracyclines, including doxycyclin, have shown to have the highest resistant rates among them. Resistance to Macrolides is also getting increased worldwide in about $5 \%$ among patients, but not as high as tetracyclines. Physicians should be aware of the resistant rates to avoid future therapeutic failure which in turn leads to increased costs.

The results suggest a need for a change in physician prescribing patterns. Various interventions, including continuing medical education, practice guidelines, and use profiling, have been used with varying success [19]. Also marketing medications by evidence-based and 
direct-to physician approaches was found to be useful in reducing poly-pharmacy and leading to appropriate utilization of medications [20].

\section{Limitations of the study}

Human error is natural source of limitation as the prescriptions were filled physically and illegibility should be considered. Generalization of the study to the whole Malaysian practices is not plausible, since it covered only USM clinics. Practices may vary from government to private clinic, and could be worse or better than USM clinics. As we have different physician practicing at our clinics, every clinic is run by two or three physicians and the patterns may describe the behavior of the clinics as a whole rather than to be specific to a particular physician. Finally, pricing policy vary according to the perspective. In our situation, the cost will be calculated from the provider point of view but not from the consumer's. Thus differences in the costs can change a lot if the perspective has been changed.

\section{CONCLUSION}

Drug expenditure for URTIs is not in consistent with the guidelines and contributed to the increased costs of the illness. Generally, antibiotics represent $58 \%$ of the total cost while antihistamines and analgesics represent 24 and $10 \%$, respectively. The over-prescribed drugs are neither consistent with the guidelines nor are they cost-effective. Prescribing antibiotics is not benign and should be thoughtfully considered and moreover, they contribute vastly to the costs of government healthcare system directly and indirectly. A guideline tailored to local needs should be created for prescription of antibiotics in URTIs and implemented to save unnecessary expenditure and avoid antibiotic-related problems.

\section{REFERENCES}

1. Miller FG, Wendler D. Direct-to-consumer advertising and physician prescribing. JAMA 2005; 294(6): 678; author reply, 678-679.

2. Wong DM, Blumberg DA, Lowe LG. Guidelines for the Use of Antibiotics in Acute Upper Respiratory Tract Infections. Am Fam Physician. 2006; 74(6): 956-966.

3. Centre for Clinical Practice at NICE (UK). Respiratory Tract Infections - Antibiotic Prescribing: Prescribing of Antibiotics for Self-Limiting Respiratory Tract Infections in Adults and Children in Primary Care.
London: National Institute for Health and Clinical Excellence (UK); 2008. National Institute for Health and Clinical Excellence: NICE Clinical Guidelines, no. 69.

4. Jewesson PJ, Ho R, Jang $Q$, Watts G, Chow AW. Auditing antibiotic use in a teaching hospital: focus on cefoxitin. Can Med Assoc J 1983; 128(9): 1075-1078.

5. Teng CL, Leong KC, Aljunid SM, Molly Cheah. Antibiotic Prescription In Upper Respiratory Tract Infections. Asia Pacific Family Medicine 2004; 3 (1-2): 38- 45

6. Yasin NM, Bahari MB , Ismail dan HIM. Antibiotic usage in pediatric respiratory tract infection. Majalah Farmasi Indonesia 2005; 16(1): 1-5.

7. Mäkelä MJ, Puhakka T, Ruuskanen O, Leinonen $M$, Saikku $P$, Kimpimäki M, Blomqvist $S$, Hyypiä $T$, Arstila P.. Viruses and bacteria in the etiology of the common cold. J Clin Microbiol 1998; 36(2): 539-542.

8. Wang EE, Einarson TR, Kellner JD, Conly JM. Antibiotic prescribing for Canadian preschool children: evidence of overprescribing for viral respiratory infections. Clin Infect Dis 1999; 29(1): 155-160.

9. Mainous AG, Hueston WJ. The cost of antibiotics in treating upper respiratory tract infections in a medicaid population. Arch Fam Med 1998; 7(1): 4549.

10. Cooper RJ, Hoffman JR, Bartlett JG, Besser RE, Gonzales R, Hickner JM, Sande MA: Principles of appropriate antibiotic use for acute pharyngitis in adults: Background. Ann Intern Med 2001; 134: 509517.

11. Piccirillo JF, Mager DE, Frisse ME, Brophy RH, Goggin A. Impact of first-line vs second-line antibiotics for the treatment of acute uncomplicated sinusitis. JAMA 2001; 286: 1849-1856.

12. Poole MD, Portugal LG. Treatment of rhinosinusitis in the outpatient setting. Am J Med 2005; 118: 45S-50S.

13. Babiker ASE. Study evaluating medication use practices and extent of drug wastage among female students at Universiti Sains Malaysia. PhD dissertation, Universiti Sains Malaysia, Penang, Malaysia 2008.

14. Rubin BK. Mucolytics, expectorants, and mucokinetic medications. Respir Care 2007; 52(7): 859-865.

15. Gagnon MA, Lexchin J.The cost of pushing pills: a new estimate of pharmaceutical promotion expenditures in the United States. PLoS Med. 2008; 5(1): e1.

16. Davey $P$, Rutherford D, Graham B, Lynch B, Malek M. Repeat consultations after antibiotic prescribing for respiratory infection: a study in one general practice. Br J Gen Pract. 1994; 44(388): 509-513.

17. Olender A, Niemcewicz M. Macrolide, lincosamide, and streptogramin B-constitutive-type resistance in Corynebacterium pseudodiphtheriticum isolated from upper respiratory tract specimens. Microb Drug Resist 2010; 16(2): 119-122.

18. Hsueh PR, Shyr JM, Wu JJ. Decreased erythromycin use after antimicrobial reimbursement restriction for undocumented bacterial upper respiratory tract infections significantly reduced erythromycin resistance in Streptococcus pyogenes in Taiwan. Clin Infect Dis 2005; 40(6): 903-905.

19. Palumbo FB, Mullins $D$. The development of direct to consumer prescription drug advertising regulation. Food Drug Law J 2002; 57(3): 423-443.

20. Lang HC, Lai MS, Chen GT. Outpatient drug expenditures and prescription policies for diseases with high cost to the National Health Insurance system in Taiwan. J Formos Med Assoc 2004; 103: 280-285. 